\title{
Baseband Radio over Fiber Aided Millimeter-Wave Distributed Antenna for Optical/Wireless Integration
}

\author{
Varghese Antony Thomas, Salman Ghafoor, Mohammed El-Hajjar, and Lajos Hanzo
}

\begin{abstract}
A Baseband Radio Over Fiber (BROF) architecture is proposed, where upto four Radio Frequency (RF) carriers can be generated, while using the heterodyne photo-detection of only two optical signals. This proposed BROF architecture has a star-like structure and it is composed of six Radio Access Units (RAUs), where data is transmitted from the Central Unit (CU) to the Base Station (BS) and from the BS to the RAU over a distance of $20 \mathrm{Km}$ and $0.3 \mathrm{Km}$, respectively, at a rate of 768 Mbps. The performance of the system supporting four carrier frequencies drops by at most $1 \mathrm{~dB}$, at a BER of $10^{-9}$, compared to conventional heterodyne photo-detection.
\end{abstract}

Index Terms-Radio over fiber, distributed antenna system, heterodyne detection, time division duplexing.

\section{INTRODUCTION}

$\mathbf{T}$ HE past decade has seen a huge increase in the number of wireless communication subscribers and the development of high-bandwidth services. Radio Over Fiber (ROF) is expected to constitute the backbone of future wireless communication systems. Baseband ROF (BROF) [1] and Analogue ROF (AROF)[2] are commonly employed optical transmission techniques in ROF communication. The ROF optical signals are transmitted over an optical network that utilises either Wavelength Division Multiplexing (WDM)[3] or Time Division Multiplexing (TDM)[4] to serve multiple Radio Access Units (RAUs). Unlike WDM, TDM requires fewer semiconductor lasers, but its employement is limited to digital optical links. The detection of these optical signals can be direct [2] or heterodyne [2],[3]. AROF employs an analogue optical link. Hence the achievable data-rates and fiber-lengths are severely limited by fiber dispersion and the optical link's non-linearity [2]. In contrast to AROF, BROF utilises a more robust digital optical link [1]. However, unlike AROF, the RAU in a BROF-based architecture performs more complex signal processing and up-conversion to Radio Frequency (RF). The need to have up-convertors in a BROF RAU can be relaxed through the use of heterodyne photo-detection [2]. However, conventional heterodyne photo-detection of two optical signals is capable of generating a signal only at a single $\mathrm{RF}$ carrier at the difference of the two optical frequencies

Manuscript received December 19, 2012. The associate editor coordinating the review of this letter and approving it for publication was T. Yioultsis.

V. A. Thomas, M. El-Hajjar, and L. Hanzo are with the School of ECS, University of Southampton, SO17 1BJ, United Kingdom (e-mail: \{vat1g10, meh, 1h\}@ecs.soton.ac.uk).

S. Ghafoor is with the National University of Sciences and Technology, Pakistan (e-mail: salman.ghafoor@seecs.edu.pk).

The financial support of the EU's Concerto Project, of the National University of Science and Technology (NUST), Pakistan, as well as that of the EPSRC, UK under the auspices of the India-UK Advanced Technology Centre and that of the European Research Council under its Advanced Fellow Grant is gratefully acknowledged.

Digital Object Identifier 10.1109/LCOMM.2013.030413.122841
[2]. In this treatise, we propose a system to overcome this limitation. We propose a Distributed Antenna System (DAS) constituted by RAUs that are fed using BROF techniques in the star-like network topology of Fig. 1d. The RAUs of each cell are connected to a Central Unit (CU) via a BS. This contribution is the outcome of the second phase of a twostage project, which is aimed at designing a multi-cellular fiber-based DAS, which is capable of significantly increasing the throughput at the cell-edges for a given transmit power, without reducing the cell-center's throughput. This involved the twin challenges of designing an efficient technique for the wireless communication over the wireless channel as well as designing a robust optical backhaul. A wireless link was designed in [5] using the architecture of Fig. 1d, where the cluster of cells was operated at four carrier frequencies. Our study used VPITransmissionMaker 8.6 (www.vpiphotonics.com) - a commercial optical simulation software.

We propose an optical fiber back-bone that is based on our novel technique that generates wireless signals at multiple RF carriers, while retaining the ability to use a digital optical link and a single optical source. Against this background, the new contributions of this paper are:

1) The proposed sytem is capable of transmiting signals at multiple RF carriers, while using heterodyne detection of only two optical signals and without any significant change of hardware components. The extent of overlap between the pulses in the two optical signals was varied, using optical delay lines, for the sake of generating multiple RF carriers.

2) The proposed system is capable of simultaneously performing photo-detection and conversion from opticalTDM to wireless Frequency Division Multiplexing (FDM). The conversion is achieved by varying the optical delay.

3) We propose the heterodyning of two pulsed optical signals that are generated from a single pulsed semiconductor laser.

Experimental proof of a similar concept was provided in [12], where an unbalanced optical modulator was used with no heterodyne detection. The proposed technique draws its motivation from optical serrodyne modulation [13], [14], which uses modulation-induced chirp for optical frequency translation.

\section{PhysicAl LAYER OF THE BASEBAND DAS}

a) Control Unit: The DL signal transmitted to each of the six RAUs has a bit-rate of $R_{D L}=128 \mathrm{Mbps}$, where the RAUs are represented by the index $k(k=0,1, . .5)$ in Fig. 1a. They are multiplexed using TDM, resulting in a total 


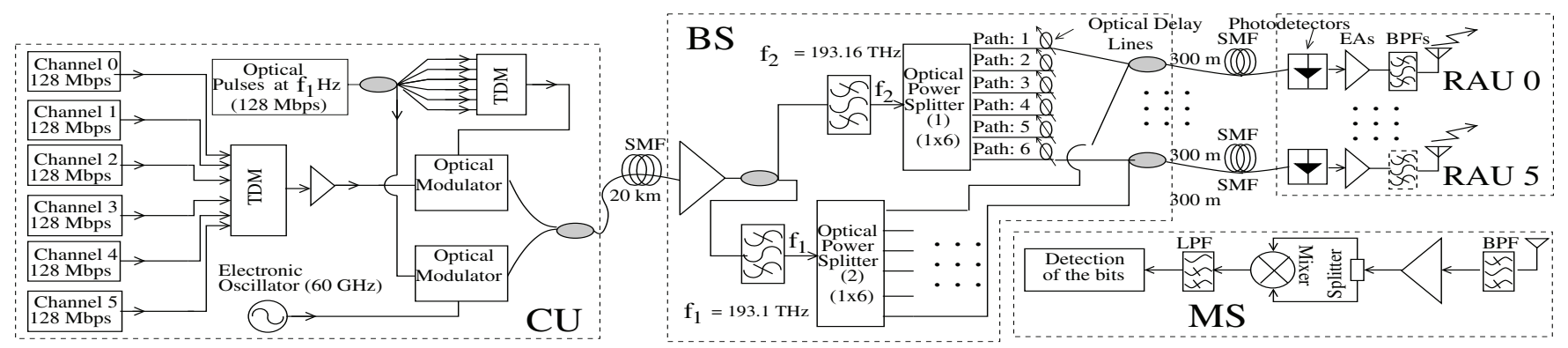

(a) Physical layer architecture of the BB DAS

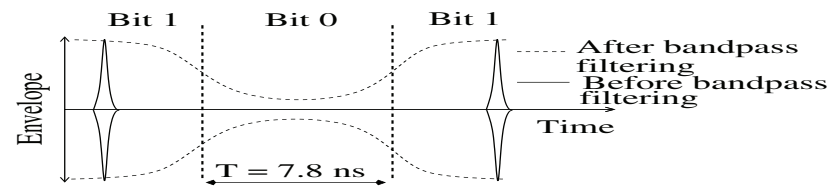

(b) Envelope of photo-detected, amplitude-modulated RF signal at RAU.

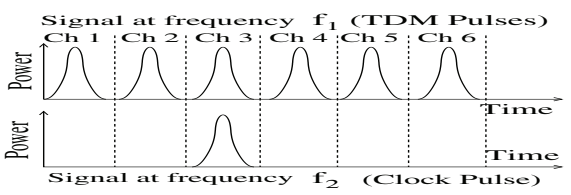

(c) Time domain pulses at $3^{\text {rd }}$ RAU (not to scale).

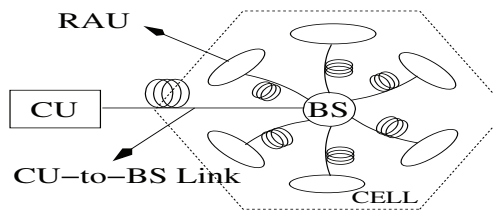

(d) Architecture of the proposed DAS

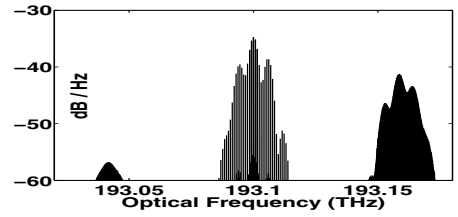

(e) Spectrum of the filtered optical signal

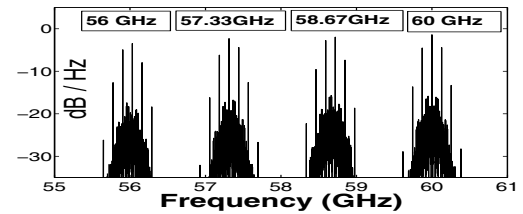

(f) Filtered photo-detected signal at the RF carriers

Fig. 1. System architecture and functioning.

bit-rate of $R_{T D M}=(6 \times 128)$ Mbps. The baseband TDM data is modulated onto a $(6 \times 128)$ Mbps pulse stream. This pulse stream is generated by time-multiplexing the output of a $128 \mathrm{Mbps}$ pulsed semiconductor laser that operates at a frequency of $f_{1}=193.1 \mathrm{THz}$ and has a pulse Full Width at Half Maximum (FWHM) $T_{F W H M}=40$ ps. Simultaneously, as shown in Fig. 1a, clock pulses are generated by modulating a $60 \mathrm{GHz}$ electronic oscillator signal onto one of the $128 \mathrm{Mbps}$ pulsed outputs from the laser. The outputs of the two optical modulators are multiplexed and transmitted to the BS over a $20 \mathrm{~km}$ Single Mode Fiber (SMF) link. This multiplexed optical signal consists of a $(6 \times 128)$ Mbps On Off Keying (OOK) signal at $193.1 \mathrm{THz}$ and of $128 \mathrm{Mbps}$ clock pulse streams at the pair of frequencies of $193.1 \mathrm{THz} \pm 60 \mathrm{GHz}$. The use of a single laser reduces complexity and cost.

b) Base Station: At the BS of Fig 1a, the incoming optical signal is amplified by an amplifier having a noise figure of $6 \mathrm{~dB}$ and then split into two paths. Optical filters having center frequencies of $f_{1}=193.1 \mathrm{THz}$ and $f_{2}=$ 193.1 $\mathrm{THz}+60 \mathrm{GHz}=193.16 \mathrm{THz}$ are used in each path for isolating the pulsed TDM signal and one of the two pulsed clock signals, respectively. These are transmitted to the RAUs through a $0.3 \mathrm{~km}$ fiber link. The six paths carrying the pulsed clock signal, as shown in Fig. 1a, include Delay Lines (DLs) to perform demultiplexing and up-conversion. The DLs ensure the selection of a particular TDM channel by ensuring that its pulses overlap with the pulses in the $128 \mathrm{Mbps}$ clock channel. The DL also controls the extent of overlap between the pulses in the clock channel and the selected channel. This in turn decides the up-converted frequency after photo-detection. Optical DLs have been widely used for time-synchronization [6] and optical signal processing [7]. Furthermore, efficient synchronization, coherent heterodyne photo-detection and up- conversion has been made possible by the availability of variable optical DLs with femtosecond sensitivity as well as by the use of a single pulsed optical source at the CU. Polarization control and time-domain overlap has been reported for optical pulses that are significantly shorter than the ones used in this paper [10], [11], with the intention of using Cross-Phase Modulation (XPM).

c) Radio Access Unit: The photo-detected RF signal consists of up-converted impulses whose temporal width equals that of the optical pulses. As shown in Fig 1a, the RF signals are amplified and then band-limited by the transmitfiltering using a Band Pass Filter (BPF) with $256 \mathrm{MHz}$ bandwidth and a Gaussian shaped time-domain response. As seen from Fig. 1b, bandpass filtering broadens these impulses. This signal is then transmitted to the Mobile Station (MS), where it is filtered, amplified and down-converted to baseband, without an oscillator, through self-mixing [8], as shown in Fig 1a. The signal is then Low Pass Filtered (LPF) and decoded.

d) Uplink Communication: The uplink (UL) RF signal is down-converted to baseband by self-mixing [8]. The 193.16 THz clock pulses do not carry OOK data and can be reused at the RAU to carry the UL baseband signal, thereby reducing the RAU complexity just like in [16]. The UL optical signals from the RAUs are sent to the BS, where they are time-multiplexed and then sent to the $\mathrm{CU}$, for direct photo-detection. Heterodyne photo-detection is not needed, since there is no up-conversion at the CU. The narrow photo-detected pulses are low-pass filtered (thereby broadening them), Time Division Demultiplexed and used for bit detection.

\section{TheORETICAL ANALYSis}


a) Time Division Demultiplexing (TDD): Let $x_{k}(t)$ be the envelope of the pulsed optical signal for the $k^{t h}$ RAU. Then, the TDM optical signal, $x_{t d m}(t)$, that is received at the RAUs and is centred at an optical frequency of $f_{1}$, can be represented as:

$x_{t d m}(t)=\sum_{k=0}^{5} x_{k}\left(t-k t_{b i t}\right) \cos \left[2 \pi f_{\text {inst } 1}\left\{t-k t_{b i t}\right\}\left(t-k t_{\text {bit }}\right)\right]$

where $f_{\text {inst } 1}\{t\}$ is the instantaneous frequency, while $t_{\text {bit }}=$ $1 / R_{T D M}$ is the multiplexing delay. The clock signal, at an optical frequency of $f_{2}$ is $x_{\text {clock }}(t)=c(t) \cos \left(2 \pi f_{\text {inst } 2}\{t\} t\right)$, where $c(t)$ is the envelope of the pulsed clock signal, while $f_{\text {inst } 2}\{t\}$ is the instantaneous frequency. As seen from Fig. 1c, channel selection is achieved by delaying the clock pulse by $k t_{\text {bit }}$ seconds to ensure that the pulses in the clock signal overlap with the pulses in the desired TDM channel. Mathematically, this is expressed as follows:

$$
\begin{aligned}
& x_{c l o c k}\left(t-k t_{b i t}-t_{o d}\right) \cdot x_{t d m}(t)=c\left(t-k t_{b i t}-t_{o d}\right) . \\
& x_{k}\left(t-k t_{\text {bit }}\right) \cdot \cos \left[2 \pi f_{\text {inst } 1}\left\{t-k t_{\text {bit }}\right\}\left(t-k t_{\text {bit }}\right)\right] . \\
& \cos \left[2 \pi f_{\text {inst } 2}\left\{t-k t_{\text {bit }}-t_{o d}\right\}\left(t-k t_{\text {bit }}-t_{o d}\right)\right],
\end{aligned}
$$

where $t_{o d}$, with $\left|t_{o d}\right| \ll t_{b i t}$, is the additional delay applied for variable up-conversion. Note that the actual delay applied to the clock would vary slightly from the theoretically calculated value, in order to account for the difference in the optical pulse propogation velocities at frequencies of $f_{1}$ and $f_{2}$.

b) Variable Up-conversion: The instantaneous frequencies $f_{\text {inst } 1}\{t\}$ and $f_{\text {inst } 2}\{t\}$ of the optical TDM signal and the clock signal are as follows:

$$
f_{\text {inst } 1}\{t\}=f_{1}+\Delta f\{t\}, f_{\text {inst } 2}\{t\}=f_{2}+\Delta f\{t\} .
$$

where $\Delta f\{t\}$ is the linear frequency chirp. This chirp is introduced by either one or both of the following factors - 1) Linear chirp due to fiber dispersion [9], 2) Additional linearchirp may be obtained by employing pulsed optical sources that are chirped. If $\tau$ is the time normalised by the bit-period in the $n^{t h}$ bit duration $(n=0,1,2 \ldots)$, then $\tau=-\frac{t_{b i t}}{2}+t-n t_{\text {bit }}$. Hence $-\frac{t_{b i t}}{2} \leq \tau<\frac{t_{b i t}}{2}, \quad \forall t$. Now, $\Delta f\{t\}=M \tau$, where $M=\left[C /\left(2 \pi T_{0}^{2}\right)\right]$ [9], [15]. Here $C$ is the chirp parameter and $T_{0}=T_{F W H M} / 1.665$. Additionally, $M$ can be adjusted by employing chirped optical sources and by varying the chirp parameter of the generated pulses. Thus, as seen in Fig. 2, the instantaneous frequency changes linearly across each pulse and it is centred around the optical carrier frequency. Hence the spectra of these optical signals are centred around the frequencies $f_{1}$ and $f_{2}$, which is also seen from Fig. 1e. The spectra become wider as the optical pulses become narrower. Time instances, which are separated by integer multiples of the bit period have the same normalised time instant. Hence we have,

$$
\Delta f\left\{t-k t_{\text {bit }}\right\}=\Delta f\{t\}=M \tau .
$$

In addition to the delay that is applied to perform channel selection, an additional overlap delay, $t_{o d} \ll t_{b i t}$, is applied to the clock signal. This results in a slight overlap mismatch between the clock pulse and the pulse in the selected channel. The chirp in the delayed clock signal is as follows:

$$
\Delta f\left\{t-k t_{\text {bit }}-t_{o d}\right\}=\Delta f\left\{t-t_{o d}\right\}=M\left(\tau-t_{o d}\right) .
$$

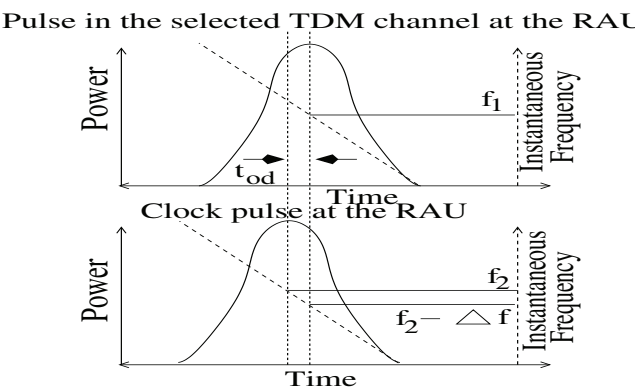

Fig. 2. Pulses at frequencies $f_{1}$ and $f_{2}\left(M<0, t_{o d}<0\right)$.

The signal generated during the heterodyne photo-detection of the TDM and clock signals at the RAU has a centre frequency, $f_{p d}$, that is equal to the derivative of the instantaneous phase difference $\Delta \phi(t)$ between the two optical signals, i.e. we have $\left.f_{p d}=(1 /(2 \pi))(d \Delta \phi(t)) / d t\right)$. Thus:

$$
f_{p d}=\frac{d}{d t}\left\{\int_{0}^{t-k t_{b i t}-t_{o d}} f_{\text {inst } 2}\left\{t^{\prime}\right\} d t^{\prime}-\int_{0}^{t-k t_{b i t}} f_{\text {inst } 1}\left\{t^{\prime}\right\} d t^{\prime}\right\},
$$

where $t^{\prime}$ is the integration variable. However, it is known from calculus that for a function $u(x), \frac{d}{d x}\left\{\int^{x} u\left(x^{\prime}\right) d x^{\prime}\right\}=u(x)$ holds. Hence the above expression of $f_{p d}^{a}$ becomes:

$$
\begin{aligned}
& f_{\text {pd }}=f_{\text {inst } 2}\left\{t-k t_{\text {bit }}-t_{o d}\right\}-f_{\text {inst } 1}\left\{t-k t_{\text {bit }}\right\} \\
& =f_{2}+\Delta f\left\{t-k t_{\text {bit }}-t_{o d}\right\}-\left(f_{1}+\Delta f\left\{t-k t_{\text {bit }}\right\}\right) \\
& =\left(f_{2}-f_{1}\right)+\left(\Delta f\left\{t-k t_{\text {bit }}-t_{o d}\right\}-\Delta f\left\{t-k t_{\text {bit }}\right\}\right) \\
& =60 \times 10^{9}-M t_{\text {od }}[\text { using equations (3) and (4)] }
\end{aligned}
$$

Hence, the centre frequency of the photo-detected signal can be varied by varying the extent of mismatch. On the other hand, conventional heterodyne detection generates RF signals at a single frequency for a given pair of optical signals.

c) TDD \& Variable Upconversion via Heterodyne Detection : The electronic signal $x_{p d}$, that is obtained at the RAU by using a photo-detector with responsivity of $r=0.9$ $\mathrm{A} / \mathrm{W}$ followed by bandpass filtering is represented as follows:

$$
\begin{aligned}
& x_{p d}(t)=b p f\left\{r \cdot\left|x_{t d m}(t)+x_{c l o c k}\left(t-k t_{b i t}-t_{o d}\right)\right|^{2}\right\}, \\
& =b p f\left\{\mid r \cdot x_{t d m}^{2}(t)+r \cdot x_{c l o c k}^{2}\left(t-k t_{b i t}-t_{o d}\right)\right. \\
& \left.\quad+2 r \cdot x_{t d m}(t) \cdot x_{c l o c k}\left(t-k t_{b i t}-t_{o d}\right) \mid\right\},
\end{aligned}
$$

where $b p f\{$.$\} is the bandpass filtering function centred at f_{p d}$ $\mathrm{Hz}$. We use Eq. (1) followed by a trignometric expansion of the third term in Eq. (6). The first 2 terms of Eq. (6) and one of the terms obtained from the trignometric expansion represent spectral components that are removed by the bandpass filter. Hence, the filtered signal can be represented as:

$$
\begin{aligned}
& x_{p d}(t)=r \cdot c\left(t-k t_{b i t}-t_{o d}\right) \cdot x_{k}\left(t-k t_{b i t}\right) \cdot \cos [\Delta \phi(t)] \\
& \approx r \cdot c\left(t-k t_{b i t}\right) \cdot x_{k}\left(t-k t_{b i t}\right) \cdot \cos [\Delta \phi(t)]\left[a s t_{o d} \ll t_{b i t}\right] \\
& \approx r \cdot \tilde{x}_{k}\left(t-k t_{b i t}\right) \cdot \cos [\Delta \phi(t)],
\end{aligned}
$$

where $\tilde{x}_{k}(t)$ carries the same OOK information as $x_{k}(t)$ but consists of approximately squared Gaussian pulses. 


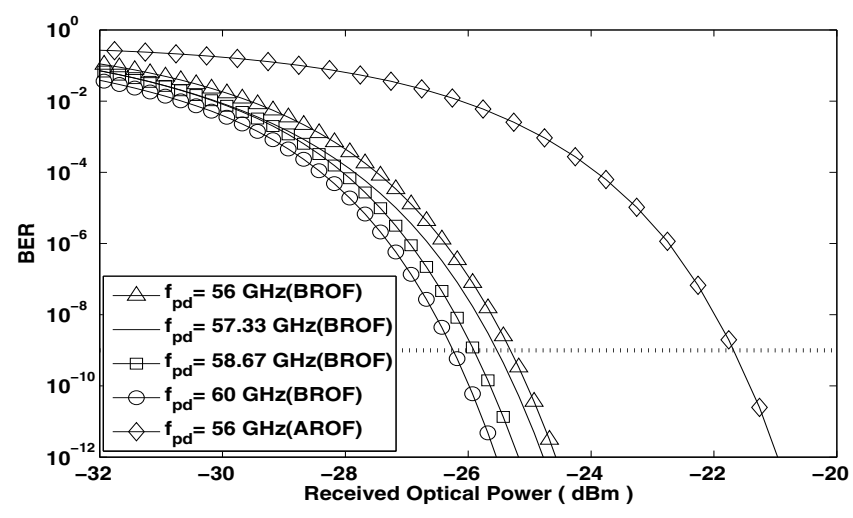

Fig. 3. BER vs. received optical power for the proposed method and AROF.

\section{System Performance}

Simulations were carried out by exploiting the fiberdispersion induced chirp. The cell was operated using signals at RF carriers of $56 \mathrm{GHz}, 57.33 \mathrm{GHz}, 58.67 \mathrm{GHz}$ and 60 GHz. These are shown in Fig. 1f. The dispersion parameter of single mode fibers at a wavelength of $1550 \mathrm{~nm}$ is $\mathrm{D}=$ $16 \mathrm{ps} / \mathrm{km}-\mathrm{nm}$ [15]. Using pulses having $T_{F W H M}=40 \mathrm{ps}$ results in a value of $C=-0.9516$ [9], which in turn gives a value of $M=-2.6241 \times 10^{20}$. Hence, from Eq. 5, $t_{o d}=3.8 \mathrm{ps}$ produces a frequency variation of $1 \mathrm{GHz}$. The tolerable deviation in the photo-detected signal's frequency from $60 \mathrm{GHz}$ is dictated by the condition that the overlap mismatch should not cause a significant Bit Error Ratio (BER) degradation and receiver sensitivity drop at BER $=10^{-9}$. The tolerable deviation in the photo-detected signal's frequency from $60 \mathrm{GHz}$ is dictated by the condition that the overlap mismatch should not cause a significant Bit Error Ratio (BER) degradation and receiver sensitivity drop at $\mathrm{BER}=10^{-9}$. The BER of conventional heterodyne photo-detection has been dealt with in chapter ten of [15]. The various factors that cause a BER penalty when compared to conventional heterodyne detection are-

1) Pulse mismatch: The detected signal is down-converted and sampled. Sampling occurs at the bit-center. There is a performance penalty, when a $2^{\text {nd }}$ delayed Gaussian pulse is multipled.

2) Bit boundary mismatch: The difference in the instantaneous optical frequency will not be equal to the target value at the bit boundaries. This, however, is negligible for very small $t_{o d}$ because the pulse amplitude at the bit boundary is negligible. Its significance, nevertheless, increases as $t_{\text {od }}$ increases.

The performance drop of the system supporting the four RF carriers is within $1 \mathrm{~dB}$, at a BER of $10^{-9}$, compared to conventional heterodyne photo-detection. Futhermore, the advantage of using a BROF architecture that employs a digital optical link in preference to the more commonly employed
AROF architecture can also be seen from Fig. 3. The severity of fiber-dispersion and the consequent sideband-cancellation, increases with the carrier frequency in analogue optical links. The BER performance of $60 \mathrm{GHz}$-band AROF links is limited by chromatic dispersion.

\section{Conclusions}

A novel technique was proposed for varying the carrier frequency of the signal, that is generated by heterodyne photodetection of 2 given optical signals. This technique varies the extent of overlap between the pulses in the 2 optical channels in order to exploit the pulse chirp. Its performance in a BROF architecture, constituted by a star-like DAS of 6 RAUs, was quantified. Also, the BER performance at the $4 \mathrm{RF}$ carriers used in the BROF system was better than that of AROF.

\section{REFERENCES}

[1] D. Novak, "Hybrid fiber radio-the application of photonic links in wireless communication systems," OFC 2009 Short Course 217.

[2] U. Gliese, S. Norskov, and T.N. Nielsen, "Chromatic dispersion in fiberoptic microwave and millimeter-wave links," IEEE Trans. Microw. Theory and Techn., vol. 44, pp. 1716-1724, Oct. 1996.

[3] T. Kuri and K. Kitayama, "Optical heterodyne detection technique for densely multiplexed millimeter-wave-band radio-on-fiber systems," IEEE J. Lightw. Technol., vol. 21, pp. 3167-3179, Dec. 2003.

[4] K. Prince, A. V. Osadchiy, and I. T. Monroy, "WIMAX radio-on-fibre in 118-km long-reach PON with deployed fibre," in Proc. 2009 European Conf. on Optical Communication, pp. 1-2.

[5] X. Xu, R. Zhang, S. Ghafoor, and L. Hanzo, "Imperfect digital-fiberoptic-link-based cooperative distributed antennas with fractional frequency reuse in multicell multiuser networks," IEEE Trans. Veh. Technol., vol. 60, pp. 4439-4449, Nov. 2011.

[6] J. Zhang, "Dual wavelength optical fiber HDTV distribution networks using self-synchronization technique and multistar topology," IEEE Trans. Consum. Electron., vol. 40, pp. 985-991, Nov. 1994.

[7] K. P. Jackson, S. A. Newton, B. Moslehi, M. Tur, C. C. Cutler, J. W. Goodman, and H. J. Shaw, "Optical fiber delay-line signal processing," IEEE Trans. Microw. Theory and Techn., vol. 33, pp. 193-210, Mar. 1985.

[8] A. Wiberg, P. Perez-Millan, M. V. Andres, P. A. Andrekson, and P. O. Hedekvist, "Fiber-optic 40-GHz mm-wave link with $2.5-\mathrm{Gb} / \mathrm{s}$ data transmission," IEEE Photon. Technol. Lett., vol. 17, pp. 1938-1940, Sept. 2005.

[9] G. P. Agrawal, Nonlinear Fiber Optics, 3rd edition. Elsevier, 2001.

[10] A. Bogris, P. Velanas, and D. Syvridis, "Numerical investigation of a $160-\mathrm{Gb} / \mathrm{s}$ reconfigurable photonic logic gate based on cross-phase modulation in fibers," IEEE Photon. Technol. Lett., vol. 19, pp. 402-404, Mar. 2007.

[11] A. Bogoni, L. Poti, R. Proietti, G. Meloni, F. Ponzini, and P. Ghelfi, "Regenerative and reconfigurable all-optical logic gates for ultra-fast applications," Electron. Lett., vol. 41, pp. 435-436, Mar. 2005.

[12] H. Chi and J. Yao, "An Approach to photonic generation of highfrequency phase-coded RF pulses," IEEE Photon. Technol. Lett., vol. 19, pp. 768-770, May 2007.

[13] A. K. M. Greenbaum and A. Wallen, "Serrodyne frequency translation using stepped modulation waveforms," IEEE Trans. Aerosp. Electron. Syst., vol. AES-10, pp. 537-538, July 1974.

[14] E. Y. I. Tomita, H. Sanjoh, and Y. Yoshikuni, "Novel method for generating multiple wavelengths by pulsed serrodyne modulation," IEEE Photon. Technol. Lett., vol. 15, pp. 1204-1206, Sept. 2003.

[15] G. P. Agrawal, Fiber-Optic Communication Systems, 3rd edition. John Wiley and Sons, 2002.

[16] S. Ghafoor, V. A. Thomas, and L. Hanzo, "Duplex digitized transmission of 64-QAM signals over a single fiber using a single pulsed laser source," IEEE Commun. Lett., vol. 16, pp. 1312-1315, Aug. 2012. 